\title{
Surgical trainee experience and opinion of robotic surgery in surgical training and vision for the future: a snapshot study of pan-specialty surgical trainees
}

\author{
Christina A. Fleming ${ }^{1,2}$. Oroog Ali $^{1,3}$. Joshua M. Clements ${ }^{1,4}$. Johnathan Hirniak ${ }^{1,5}$. Martin King ${ }^{1,6}$. \\ Helen M. Mohan ${ }^{1,7}$. Deirdre M. Nally ${ }^{1,2}$. Josh Burke ${ }^{1,8}$ on behalf of The Association of Surgeons in Training (ASIT)
}

Received: 11 August 2021 / Accepted: 21 November 2021 / Published online: 26 November 2021

(C) The Author(s), under exclusive licence to Springer-Verlag London Ltd., part of Springer Nature 2021

\begin{abstract}
Background: Robotic surgery is well established across multiple surgical specialities in the United Kingdom (UK) and Republic of Ireland (ROI). We aimed to elucidate current surgical trainee experience of and attitudes to robotic surgery in a surgical training programme across the UK and ROI to determine the future role of robotic surgery in international surgical training programmes. Methods: A pan-specialty trainee cross-sectional study was performed on behalf of the Association of Surgeons in Training (ASiT) using mixed-methodology. Round 1: a digital questionnaire was disseminated to all ASiT members. Round 2: 'live-polling' was performed prior to and following the Robotic Surgery plenary session convened at the ASiT 2020 International Conference (Birmingham). Data analysis was performed using a combination of quantitative and qualitative methods. Results: Three hundred and four responses were analysed ( $n=244$ digital questionnaire, $n=60$ live-polling). Overall, $73.8 \%(n=180)$ of trainees would value greater access to robotic surgery training. $73.4 \%(n=179)$ believed that robotic surgery was important for the future of their desired specialty and $77.2 \%(n=156)$ believed it should be incorporated into formal surgical training. Qualitative analysis identified that trainees believe that robotic training should have a formal role in surgical training. Perceived disadvantages of robotic surgery experience in surgical training included expense and the current impact of consultant robotic learning curves on training. Conclusion: Current surgical trainees desire greater access to robotic surgery in surgical training. Robotic surgery is developing an increasing role in current surgical practice and it is important that it is introduced in a timely, evidence-based fashion to surgical trainees at an appropriate stage of training.
\end{abstract}

Keywords Robotics $\cdot$ Robotic surgery $\cdot$ Surgical training

\section{Introduction}

Previous Communication to Society or Meeting ASiT Conference 2021 (virtual)

Christina A. Fleming

christina.fleming49@gmail.com

1 Association of Surgeon in Training Council, London, UK

2 Department of Colorectal Surgery, Mater Misericordiae University Hospital, Dublin 7, Dublin, Ireland

3 Queen Elizabeth Hospital, Gateshead, UK

4 Belfast City Hospital, Belfast, Northern Ireland

5 St George's University of London, London, UK

6 Causeway Hospital, Coleraine, Northern Ireland

7 St. Vincent's University Hospital, Dublin 4, Dublin, Ireland

8 St. James's Teaching Hospital Trust, Leeds, UK
Robotic surgical systems are rapidly entering international clinical practice [1]. These systems provide superior platforms with 3D visualisation, reduced tremoring and endowristed instruments all supporting the surgeons' ability to improve operative precision with the ultimate aim to improve patient outcomes. Early evidence supports that the ergonomics of robotic surgery are more favourable for the operating surgeon than laparoscopy [2]. Reduced fatigue and musculoskeletal strain may improve concentration and performance; however, how this may impact on clinical or operative outcomes still requires investigation. Robotic surgical systems have been in development since 1950s; however, they were more broadly adopted in 2000 when Intuitive Surgical entered the clinical market with the daVinci system (California, USA). It was a single player for almost 20 years until 
the market introductions of Transenterix (Asensus Surgical, US), Versius (Cambridge, UK) and multiple other platforms currently in development [3]. In 2019, it was reported that over 5000 daVinci systems were installed worldwide and over 6 million procedures performed across multiple surgical specialities. Robotic practice is now established with varying penetrance across most of the surgical specialities [4].

The adoption of robotic surgical systems has been criticised due to high costs, significant training requirements, lack of standardised curriculae and quality assurance procedures [5]. Robotic surgical systems require the learning of new skills in docking, instrument use and in-console operating, particularly with adaptation to the loss of haptic feedback present in laparoscopic or open surgery. Industry partners provide both simulated training and live operative proctoring to surgeons on commencing robotic surgery programmes; however, to date, there remains no formal mandatory curriculum for robotic surgical training across specialities independent of industry [6]. There are however a number of specialty organisations that have published guidance and recommendations on how robotic surgery and robotic surgical training should be delivered and quality assured [7-10]. An example is the European Academy of Robotic Colorectal Surgery (EARCS) which provided a formal, optional training scheme for colorectal surgeons that included a combination of didactic teaching, simulation, cadaveric work, live operating with proctoring and blinded video assessment. Standardised guidelines describing how specific procedures should be performed robotically have also been produced [7, 11].

As a rapidly evolving area of surgical practice, this has direct relevance to current surgical trainees across all specialities [12]. There is currently no international consensus on the introduction of a standard training pathway or curricula for robotic surgery in surgical training. The current surgical training curricula in the UK and Ireland also do not address robotic surgery and it is unclear what its current role is in surgical training apart from anecdotal reports [13]. The aim of this study was to investigate current surgical trainee experience of robotic surgery across the UK and Ireland and establish current pan-speciality surgical trainee views on the role of robotic surgery in both their current and future surgical practice.

\section{Methods}

A pan-specialty trainee cross-sectional study was performed on behalf of the Association of Surgeons in Training (ASiT) using mixed-methodology in keeping with STROCSS guidelines and Standards for Reporting Qualitative Research [14, 15]. Initially, a digital questionnaire was disseminated to ASiT members in early 2020.
Subsequently, 'live-polling' was performed prior to and following a plenary session on robotic surgery convened at the ASiT 2020 International Conference (Birmingham). ASiT is a pan-specialty professional and educational body. ASiT works to promote excellence in surgical training for the benefit of junior doctors and patients alike in the UK and Ireland. ASiT was founded in 1976 and is independent of the National Health Service (NHS), Surgical Royal Colleges and Specialty Associations. ASiT currently represents over 3,500 surgical trainees across all ten surgical specialties.

\section{Round 1: Questionnaire design and dissemination}

A bespoke questionnaire was designed by the study steering group combining both quantitative and qualitative questions. Data were sought to identify current surgical trainee experience of robotic surgery and views and expectations of the role of robotic surgery in the future including the role in training in general and within chosen specialty. The survey consisted of free text (for qualitative analysis), binomial and multinomial responses. Following initial design, the questionnaire was distributed and piloted within ASiT's executive committee followed by ASiT's elected council, comprising over sixty surgical trainees across all stages of training and specialty to ensure content and face validity. The feedback received was used to further refine the question items both in terms of content and wording in order to remove ambiguity and ensure question neutrality.

The questionnaire was distributed through the ASiT members' mailing list. Participant demographics were recorded including gender and stage of training, classified as follows: higher surgical trainee (HST) - trainee enrolled in a 'specialty training programme', following competitive application. HST lasts up to 6 years and provides dedicated training in one of the ten defined surgical specialities (general surgery, trauma and orthopaedics, urology, paediatric surgery, otorhinolaryngology, plastic surgery, oral and maxillofacial surgery, cardiothoracic surgery, neurosurgery and vascular surgery). Core surgical trainee (CST)—doctors wishing to pursue a career in surgical specialities appointed through a UK-wide national competitive selection process into a "Core Surgical Training" programme lasting 2 years (this training may be generic or themed around a particular surgical speciality); Foundation year doctor (FY) - participant in a 2-year generic postgraduate programme following completion of undergraduate degree; Medical students. Current hospital work-setting was also recorded as University Teaching Hospital or District General Hospital. 


\section{Round 2: International conference live-polling}

A plenary session entitled Optimisation through Robotics was convened during the ASiT International Conference 2020 (March, Birmingham) involving pan-specialty lectures from experts in robotics with specific emphasis on surgical training and patient outcomes following robotic surgery. Delegates were invited to answer the following questions via 'live-polling' before and after the session: (1) what is the first word you think of when you hear 'robotic surgery'?; (2) what are the advantages of robotic surgery?; (3) what are the disadvantages of robotic surgery?; (4) should robotic surgery be incorporated into surgical training?; (5) how should robotic surgery training be delivered? The session was moderated using the web platform Slido (https://www.sli.do). Questions 1-3 captured responses visually in word clouds. The size and colour intensity of each word increased with its prevalence in summarised data. Question four identified multiple choice responses and Question five gathered freetext responses. The aim was to identify if further education on the potential benefits of robotic surgery would influence views on the role of robotic surgery both in training and in the future of surgery.

\section{Data analysis}

Quantitative data analysis was performed using SPSS (IBM), version 24. Data were analysed for the entire group and further analysed according to three subgroups as follows: Group 1-Medical student, foundation year, core trainee, SHO (non-training); group 2-surgical trainee ST3-5; group 3-ST6-8, SR (senior registrar non-training), post-certification of completion of training (CCT) fellow. Qualitative analysis, using an inductive thematic analytical approach, was performed on the free text comments provided by survey respondents. To facilitate and support the coding process, the survey was imported into Nvivo 12 for Windows (QSR International Pty Ltd., Australia). DN generated an initial coding framework. The steering committee collaboratively reviewed and reflected on these initial codes, and considered preliminary themes. Through further reflexive discussion, these themes were refined, defined and named. Illustrative examples were selected for inclusion in this manuscript.

\section{Results}

\section{Participant demographics}

Two hundred and forty four responses were received to the online questionnaire. $37.3 \%(n=91)$ identified as female and $59.8 \%(n=146)$ as male. Academic trainees comprised $13.9 \%(n=34)$, less than full time (LTFT) trainees
$4.5 \%(n=11)$ and $2 \%$ military $(n=5)$. Over fifty percent of trainees currently worked in a University Hospital (59\%, $n=144)$. The most popular planned future specialties and subspecialties were general surgery at $26.2 \%(n=64)$, colorectal surgery at $23.8 \%(n=58)$ and urology at $12.7 \%$ $(n=31)$. A full breakdown of level of experience and specialty interest is available in Table 1. Group one (most junior) comprised $34.0 \%(n=83)$ of the trainee cohort, group

Table 1 Study demographics (age, gender, level of training, current hospital, specialty interest)

\begin{tabular}{|c|c|c|}
\hline$N=244$ & $\%$ & $N$ \\
\hline \multicolumn{3}{|l|}{ Gender } \\
\hline Female & 37.3 & 91 \\
\hline Male & 59.8 & 146 \\
\hline Not-specified & 2.9 & 7 \\
\hline \multicolumn{3}{|l|}{ Training programme } \\
\hline Academic & 13.9 & 34 \\
\hline Military & 2 & 5 \\
\hline LTFT & 4.5 & 11 \\
\hline \multicolumn{3}{|l|}{ Grade } \\
\hline Medical student & 3.3 & 8 \\
\hline Foundation year & 8.2 & 20 \\
\hline Core surgical training & 18.0 & 44 \\
\hline SHO (non-training) & 4.1 & 10 \\
\hline ST3-5 & 24.2 & 59 \\
\hline ST6-8 & 30.7 & 75 \\
\hline SR (non-training) & 3.7 & 9 \\
\hline Post-CCT fellow & 7.8 & 19 \\
\hline \multicolumn{3}{|l|}{ Current training setting } \\
\hline University Hospital & 59 & 144 \\
\hline District General Hospital & 38.9 & 95 \\
\hline Other & 2 & 5 \\
\hline \multicolumn{3}{|l|}{ Current specialty } \\
\hline Cardiothoracic surgery & 0.4 & 1 \\
\hline Colorectal surgery & 23.8 & 58 \\
\hline Core Surgical training & 5.3 & 13 \\
\hline Foundation training & 5.7 & 14 \\
\hline General surgery & 26.2 & 64 \\
\hline Hepatobiliary surgery & 2.1 & 5 \\
\hline Oral maxillofacial surgery & 1.2 & 3 \\
\hline Neurosurgery & 3.3 & 8 \\
\hline ENT & 1.2 & 3 \\
\hline Out of programme & 3.7 & 9 \\
\hline Paediatric surgery & 0.4 & 1 \\
\hline Plastic surgery & 1.2 & 3 \\
\hline Trauma and orthopaedic surgery & 4.9 & 12 \\
\hline Upper GI surgery & 7.4 & 18 \\
\hline Urology & 12.7 & 31 \\
\hline Vascular surgery & 1.2 & 3 \\
\hline
\end{tabular}


two $23.8 \%(n=58)$ and group three (most senior) $42.2 \%$ $(n=103)$.

\section{Current access and experience}

Table 2 comprehensively summarises current trainee access to and experience of robotic surgery. Overall, $73.8 \%$ $(n=180)$ of trainees would value greater access to robotic surgery training as it is perceived to have an increasingly important role in the future provision of surgical care. Currently, only $13.5 \%(n=33)$ of trainees report that robotic surgery training is available in their training region. Interestingly, $14.3 \%(n=35)$ of trainees believed that robotic surgery may have a negative impact on their training, a topic that is further explored in qualitative analysis. This was greatest for trainees at level ST3-5.

\section{Attitudes and the future}

Trainees were asked to describe one word that they believed best described robotic surgery and these findings are summarised in Fig. 1. In this word cloud, we can see the trainees felt most strongly that robotic surgery is important for the "future" with other prominent themes including innovation, expensive, precision and advanced. In Table 2, surgical trainee attitude to robotic surgery is summarised. $73.4 \%(n=179)$ of trainees believed that robotic surgery was important for the future of their desired specialty and that robotic surgery training should be delivered through a combination of simulation $(70.5 \%, n=172)$, cadaveric training $(58.2 \%, n=142)$, didactic and hands on courses $(54.4 \%$, $n=133)$ and live operating time as first assistant (52.5\%, $n=128)$ and live operating console time $(55.7 \%, n=136)$.

Over $70 \%$ of trainees believed that robotic surgery is important in current training, but $45.1 \%(n=110)$ believed not in the current form it is delivered and this was consistent among all levels of training experience: group one $39.8 \%$ $(n=33)$, group two $55.9 \%(n=33)$, group $342.7 \%(n=44)$. $77.2 \%(n=156)$ of trainees who responded felt that robotic surgery should be incorporated into formal surgical training programmes and $12.4 \%(n=25)$ were undecided. It was most commonly reported that such robotic surgery training should be delivered monthly $(25.8 \%, n=63)$ or at least every few months $(29.9 \%, n=73)$. Trainees believed that robotic surgery be funded by a combination of government $(78.3 \%, n=191)$, industry $(61.5 \%, n=150)$, the training hospital $(52.1 \%, n=127)$ and to a lesser degree the training programme $(30.7 \%, n=75)$.

\section{Qualitative analysis}

On qualitative analyses, three major themes emerged from the free-text comments provided by respondents: (1) benefits and limitations for patients and healthcare; (2) impact of consultant learning curve; (3) impact on surgical training. Trainees reported both positive and negative impacts of robotic surgery in current practice. Trainees referred to robotic surgery being 'an advancement of surgical science,' 'innovative' and 'the future'. More widespread adoption was considered, by some, to be, 'inevitable'. Perceived benefits included improved technical performance and superior patient outcomes. Proficiency was considered a competitive advantage for consultant appointment. The perceived negative impact of robotics may be summarised as trainees identifying disadvantages of robotic surgery in clinical practice including the expense (both direct and indirect costs) and the additional time required to complete robotic operations were cited. Some did not believe that the currently published outcomes justify these expenses or the opportunity costs.

Regarding the impact of the consultant learning curve, trainees reported reduced training opportunities when consultants were still gaining experience. It was suggested that favourable training cases were performed by consultants and on occasion, trainees were required to function as first assistants to facilitate this and as a result missed out on training in the robotic case and other cases running simultaneously: "It has made consultants focused on improving their numbers and experience, with good reason, but leaves trainees out of the conversation".

This was compounded in centres where there was a robotic fellow. This led into the third theme of impact on surgical training. Junior trainees had concerns that robotic surgery impacts upon the acquisition of basic surgical skills while more senior trainees who do not anticipate a robotic practice are concerned that robotic surgery reduces their exposure to open and laparoscopic operations which are required for completion of surgical training competencies.

\section{Live-polling data}

There were sixty responses to the live-polling session for overall analysis and the main word cloud findings are summarised in Fig. 2. When questioned on the first word that they thought about when hearing the phrase "robotic surgery", prior to delivery of the session the most common words were "future", "expensive", "innovation" and "futuristic". Following the session "future" remained as the most common word with less emphasis on expensive and greater emphasis given to "urology", "precision" and "standard". Prior to the session, the main advantage of robotic surgery was described as "precision" and following the session, this was expanded to include "precision", "ergonomics", "access" and "surgeons health". The initial disadvantages were identified as "expensive" and "cost" and subsequently "training" gained greater emphasis with "expensive", "cost" and "access" also frequently reported. 
Table 2 Current surgical trainee access to, experience of and attitudes to robotic surgery and robotic surgery training

Access and experience

\section{N}

Do you have access to robotic surgery training in your hospital?

\begin{tabular}{lll} 
No & 152 & 62.3 \\
Unsure & 40 & 16.4 \\
Yes (predefined times) & 20 & 12 \\
Yes (whenever I chose) & & \\
Do you have access to robotic surgery training in your training region? & 92 \\
No & 99 & 37.7 \\
Unsure & 22 & 11 \\
Yes (predefined times) & 40.6 \\
Yes (whenever I choose) & 9.0 \\
\hline Attitudes & 4.5 \\
\hline
\end{tabular}

Is robotic surgery important for the future of your specialty?

Yes, for majority of cases

Yes but only for select cases

Yes, but requires refinement

No, not supported by evidence

No, not cost effective

How should robotic surgery training be delivered?

Simulation

Cadaveric Training

Didactic and hands on courses

Live Operating (first assist)

Live operating (console time)

All Groups

Group 1

Group 2

Group 3

$N(\%)$

$N(\%)$

$N(\%)$

Is robotic surgery important in current training?
No response

No

Yes (but not in current form)

39 (16)

33 (13.5)

$110(45.1)$

$62(25.4)$

Yes (it should continue as is)
Has robotic surgery negatively impacted on your training?

$\begin{array}{ll}\text { No response } & 25(10.2) \\ \text { No } & 102(41.8) \\ \text { Not applicable } & 40(16.4) \\ \text { Unsure } & 42(17.2) \\ \text { Yes } & 35(14.3)\end{array}$

35 (14.3)
21 (25.3)

11 (13.3)

33 (39.8)

18 (21.7)

11 (13.3)

33 (39.8)

17 (20.5)

12 (14.5)

$10(12.0)$
$N(\%)$

4 (6.8)

8 (13.6)

33 (55.9)

14 (23.7)

2 (3.4)

22 (37.3)

7 (11.9)

16 (27.1)

12(20.3)

43 (78.2)

4 (7.3)

8 (14.5)

25 (12.4)

How often should robotic surgery training be delivered in formal training?

$\begin{array}{ll}\text { Annually } & 25(10.2) \\ \text { Every few months } & 73(29.9) \\ \text { Monthly } & 63(25.8) \\ \text { Weekly } & 31(12.7) \\ \text { I don't think it should be introduced } & 13(5.3)\end{array}$

7 (11.3)

49 (79.0)

$6(9.7)$

$4(6.5)$

$24(38.7)$

$23(37.1)$

$7(11.3)$

4 (6.5)
6 (10.9)

19 (34.5)

16 (29.1)

$11(20.0)$

$3(5.5)$
15 (14.6)

14 (13.6)

44 (42.7)

30 (29.1)

13 (12.6)

47 (45.6)

16 (15.5)

14 (13.6)

13 (12.6)

64 (75.3)

11 (12.9)

10 (11.8)

15 (17.0)

30 (34.1)

24 (27.3)

13 (14.8) $6(6.8)$ 
Table 2 (continued)

\begin{tabular}{|c|c|c|c|c|}
\hline & $\begin{array}{l}\text { All Groups } \\
N(\%)\end{array}$ & $\begin{array}{l}\text { Group } 1 \\
N(\%)\end{array}$ & $\begin{array}{l}\text { Group } 2 \\
N(\%)\end{array}$ & $\begin{array}{l}\text { Group } 3 \\
N(\%)\end{array}$ \\
\hline \multicolumn{5}{|c|}{ Who should fund robotic surgery training? } \\
\hline Training Hospital & $127(52.1)$ & $47(56.6)$ & $31(52.5)$ & $49(47.6)$ \\
\hline Training Programme & $75(30.7)$ & $28(33.7)$ & $11(18.6)$ & $36(35.0)$ \\
\hline Industry & $150(61.5)$ & $55(66.3)$ & $36(61.0)$ & $59(57.3)$ \\
\hline Government & $191(78.3)$ & $66(79.5)$ & $43(72.9)$ & $82(79.6)$ \\
\hline
\end{tabular}

Group 1: medical student, foundation year, core trainee, SHO (non-training). Group 2: ST 3-5. Group 3: ST6-8, SR (non-training), post-CCT fellow

Fig. 1 Trainee views on the one word that best describes robotic surgery

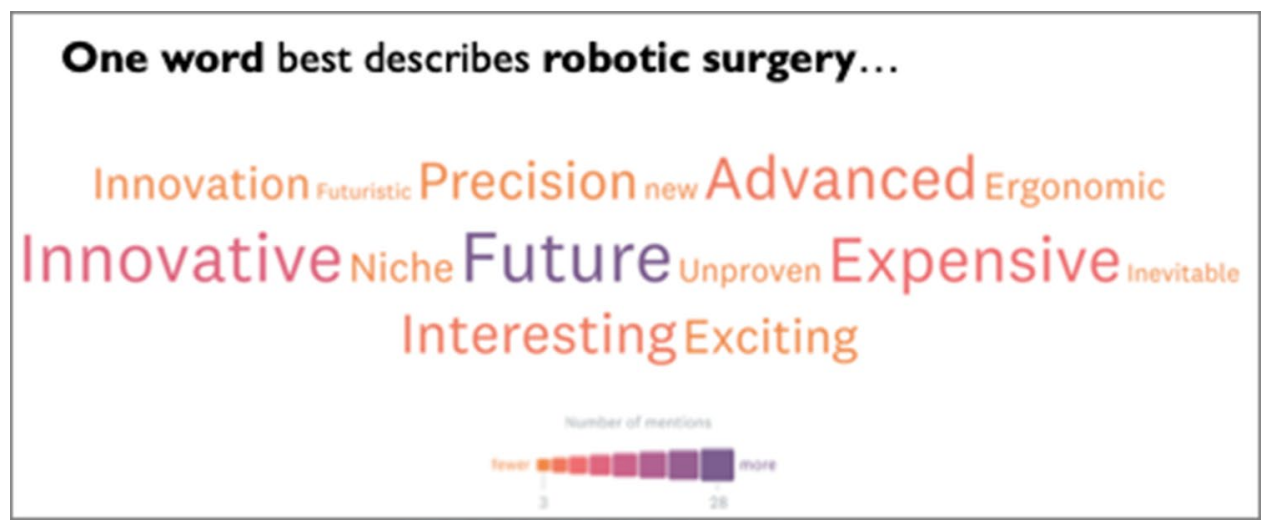

\section{Discussion}

Robotic surgery offers a more precise surgical platform with perceived benefits for both patients and surgeons [1, $2,16]$. In this study, less than $12 \%$ of current surgical trainees reported having robotic surgery training opportunities in their current hospital and three quarters of current surgical trainees desire greater access to robotic surgery training in general, preferably as part of formal surgical training. Some potential inhibitors of surgical training were described including lost training opportunities in open and laparoscopic surgery in favour of robotic surgery. Loss of opportunities to develop basic surgical skills for more junior trainees was also described and the impact of a prevalent consultant learning curve as robotic surgery is still in relative infancy. Live-polling following delivery of a plenary session on robotic training resulted in recognition of expanded potential advantages and disadvantages of robotic surgery for patients and surgeons following informative, evidence-based lectures. As we identified in this study, surgical trainees' experience of and access to robotic surgery is limited in clinical practice, so delivery of educational sessions is important.

Robotic surgical systems can offer advantages in surgical training. The $3 \mathrm{D}$ visualisation allows better visualisation of tissue planes and anatomical structures [17]. The dual-console pilot/co-pilot set-up also allows a smooth method for transfer of instruments and on screen instruction from trainer to trainee and vice versa [18]. As previously outlined, there is a lack of mandatory curriculum for robotic surgery training across any specialities independent of industry partners. However, there are specialty organisations that recommend and provide guidance on how robotic surgery training should be delivered [7-10]. Recognising the benefits of blended educational methods for learning new surgical techniques, surgical trainees in this study also reported that the desired method for delivery of robotic surgical training should encompass a combination of simulation, cadaveric and live operating with supervision and appropriate proctoring. Trainees also expressed that this should be funded through a combination of national, regional and local programmes through a variety of funding sources. Currently, there is no reference to robotic surgery within formal surgical training curricula in the UK and Ireland and this is required to ensure appropriate mapping of funding [13].

While there is a desire for greater access to robotic training among surgical trainees, it is important to discuss elements of robotic surgery that may have a perceived negative impact on surgical training overall. Of the minority of surgical trainees that did have experience of robotic surgery in 


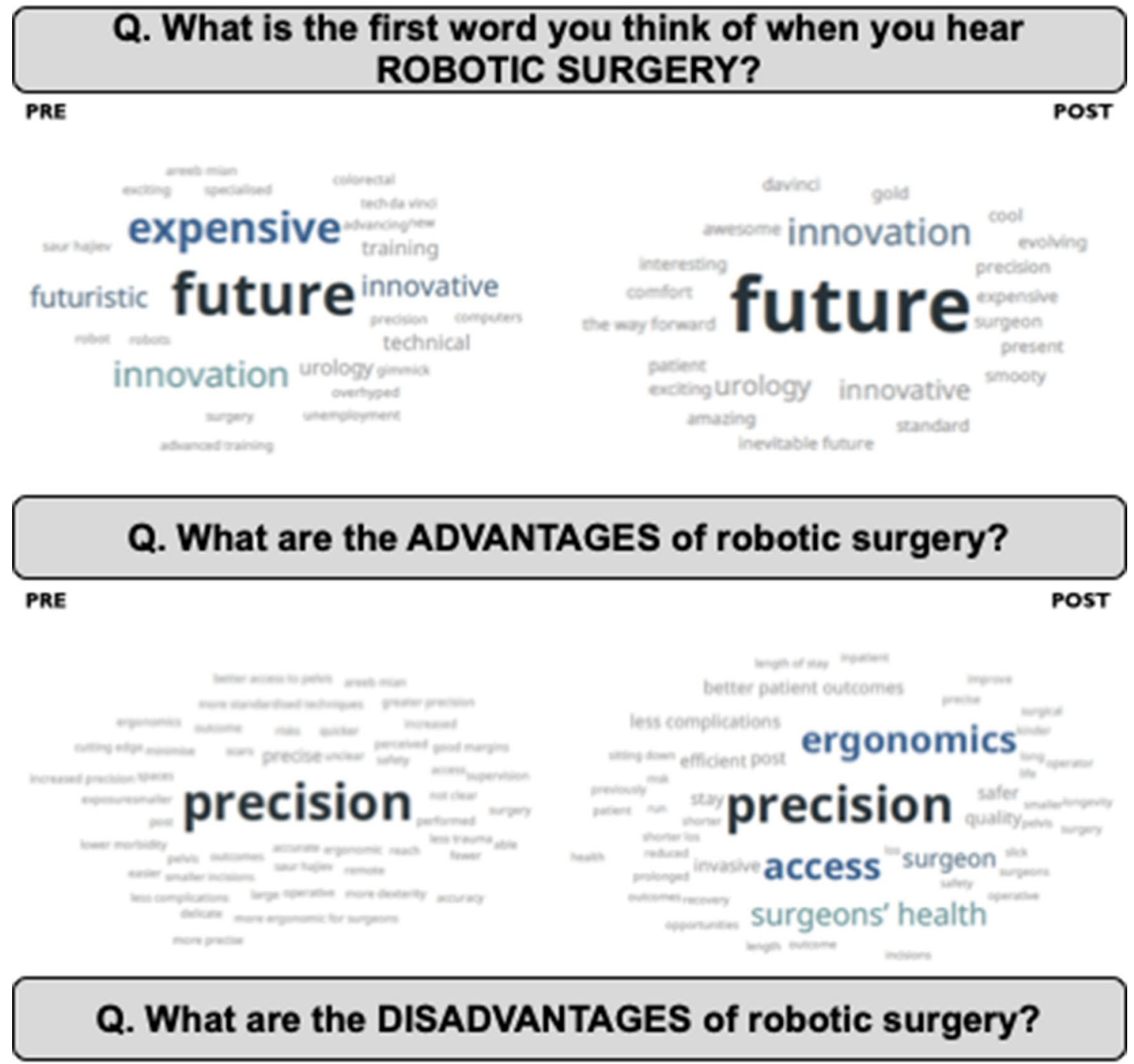

PRE

POST
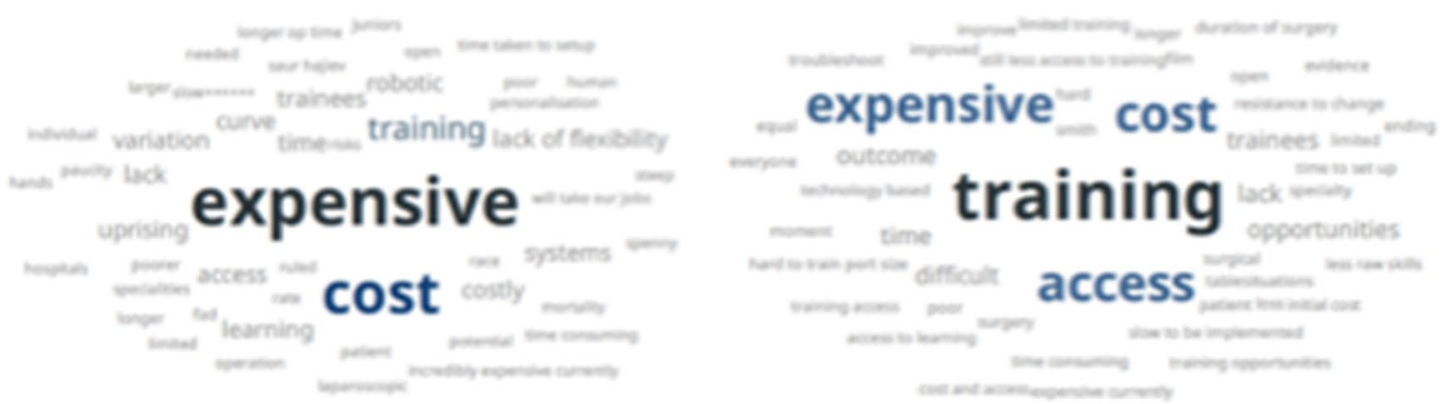

Fig. 2 Word cloud summaries of live-polling data pre- and post-plenary session

training, 14\% reported that it may have negatively impacted on their surgical training. This affected trainees of grade ST3-5 the most. In these training years, trainees begin to gain experience in resectional surgery for example in gastrointestinal surgery in both the elective and emergency setting.
Being able to perform an open resection in the emergency setting is a key learning goal during this period and this learning curve is achieved by performing a certain volume of supervised resections in the elective setting as training cases. Trainees reported that the prevalent consultant learning 
curve that still exists in robotic surgery reduced their hands on operative experience of robotic surgery and opportunities to gain experience in open and laparoscopic resections. But as consultant adoption of robotic techniques becomes more widespread, opportunities to incorporate robotic surgical training into surgical training will increase. The authors suggest that robotic surgery can offer different training benefits at different stages of training and can have a role at all stages of training. In very early years of training, basic surgical and laparoscopic skills can be developed through port insertion and acting in the role of first assistant. In intermediate stages of training, commencing console work can facilitate trainees develop basic minimally invasive surgical skills that are also transferable to laparoscopy, e.g. identifying surgical planes and landmarks for vascular ligation and in senior years putting all stages of the procedure together with appropriate graded autonomy.

The learning curve associated with robotic surgery is dependent on a number of factors. It first appears that it may depend on the institutional approach to introduction of a robotic surgery programme. As a result, some institutions have reported initial learning curves to be as high as 75 cases; however, this can be reduced to less than 30 when the robotic operative team is more experienced $[19,20]$. It is therefore expected that the impact of the consultant learning curve will reduce with increasing use of robotic surgery. For example, the most recent 2020 National Bowel Cancer Audit (NBCOA) report identified that the number of robotic colorectal cancers performed annually has doubled from 2015/2016 to 2018/2019 period and the number of surgeons performing robotic resection increased by almost $40 \%$ [21]. In addition, in 2015 almost $70 \%$ of radical prostatectomy are performed robotic-assisted reflecting increasing use of robotic surgery in urology [22]. With rapid expansion of robotic surgery provision, it is important that the learning curve is actively addressed to avoid the excessive learning curve durations that were experienced with initial introduction of laparoscopic surgery [23].

The use of a dual console in robotic surgery systems also offers a unique facility for surgical training and to reduce learning curves as two surgeons can operate together [19]. The pilot/co-pilot set-up allows a smooth method for transfer of instruments and screen directioning, supervision and proctoring. Use of a dual console is also a safe and effective operative method with no significant difference identified in consultant outcomes when compared to trainee outcomes with appropriate consultant supervision [18-20]. The dualconsole system simplifies the proctoring system substantially and may in time lead to trainees getting increased operative exposure even in the presence of consultant learning curves through parallel training. It is important to consider robotic surgery in the broad context of strategic planning for the future of healthcare. Future clinicians will inevitably manage patients who have undergone or will undergo robotic surgery. It will therefore be essential for clinicians to have a basic understanding of robotic assisted surgery, benefits and possible complications in order to better inform patients. The authors suggest that medical schools could incorporate a requirement for medical students to observe a robotic procedure during surgical placement to ensure that students have a basic understanding of the process involved and understand the role the surgeon plays in operating the robotic system [24]. This may have an added benefit of increasing interest in a surgical career.

A criticism of robotic surgery was suggested to be the financial investment required for capital and consumable costs associated with robotic surgery systems [5]. This has been a challenge to date but with the expansion of the market and more systems coming into the market, competition should dictate more competitive costs. Furthermore, some centres have shown cost-effectiveness can be balanced to favour robotic surgery when the operative learning curve has been overcome, operating theatre efficiency obtained and fewer complications achieved compared to open or laparoscopic surgery [25]. Interestingly, in a pandemic era, there is emerging evidence that robotic surgery systems may offer a "shielding layer" by reducing the amount of operative time and number of operative team members required to be in contact with potentially affected patients [26]. It is also important to continue to embrace innovation in surgical practice to ensure continued improvement in quality of surgical care and ultimately patient outcomes.

There are some limitations to this study. As the questionnaire was disseminated via a mailing list, it is difficult to report an exact response rate as it is unclear exactly how many trainees accessed and did not respond. Furthermore, robotic surgery enthusiasts are possibly more likely to engage with completing a questionnaire on robotic surgery. These data represent a cross-sectional snapshot of current trainee opinions which may be significantly impacted by experience or lack of experience of robotic surgery to date. It should also be noted that the data are skewed to senior trainees with close to $70 \%$ of respondents at a level of ST3 or higher and skewed toward specialties in which robotic surgery is well established (almost $40 \%$ of respondents identify a specific interest in Colorectal or Urology). Nonetheless, it presents trainee reported data across all ten surgical specialties and varying levels of experience in surgical training.

In conclusion, current surgical trainees desire greater access to robotic surgery in surgical training. While there are some concerns about the negative impact of certain aspects of robotic surgery on surgical training, it is expected that these will reduce as experience in and widespread use of robotic surgery increases. The COVID-19 pandemic has forced a step change in the way we work and communicate across the surgical specialities and has propelled the 
digitalisation of surgical training [27]. Robotic surgery is one large element of this and it must be ensured that it is introduced in a timely, evidence-based fashion to surgical trainees at an appropriate stage of training. Following the move to competency-based training curricula, this should be a dynamic process and one that will vary between specialties and training grades to future proof surgical training.

Authors' contributions CAF, study design, survey preparation, data analysis, manuscript preparation. OA, study design, survey preparation, data analysis, manuscript preparation. JMC, study design, data analysis, manuscript preparation. JH, study design, data analysis, manuscript preparation. MK, study design, data analysis, manuscript preparation. HMM, study design, data analysis, manuscript preparation. DMM, study design, qualitative data analysis, manuscript preparation. JB, study design, manuscript preparation.

Funding No funding was obtained to complete this study.

\section{Declarations}

Conflict of interest ASiT has developed an educational partnership with CMR Surgical which commenced following completion of this study.

\section{Ethical approval Not required.}

Data availability Data are available from the corresponding author on request.

Code availability Not applicable.

Consent to participate All participants consented to participating in this survey.

Consent for publication All authors have reviewed this manuscript and approved its progression for consideration for publication.

\section{References}

1. Sheetz KH, Claflin J, Dimick JB (2020) Trends in the adoption of robotic surgery for common surgical procedures. JAMA Netw Open 3:e1918911

2. Dalager T, Jensen PT, Eriksen JR, Jakobsen HL, Mogensen O, Søgaard K (2020) Surgeons' posture and muscle strain during laparoscopic and robotic surgery. Br J Surg 107(6):756-766. https://doi.org/10.1002/bjs.11394

3. Nof SY (1999) Handbook of industrial robotics, 2nd edn. Wiley, Hoboken, pp 3-5

4. Intuitive Surgical Inc. 2019. Annual reported. Available at: https://www.annualreports.com/Company/intuitive-surgicalinc. Accessed on 19 Dec 2020

5. Lanfranco AR, Castellanos AE, Desai JP, Meyers WC (2004) Robotic surgery: a current perspective. Ann Surg 239(1):14-21. https://doi.org/10.1097/01.sla.0000103020.19595.7d

6. Intuitive Surgical Inc, Learning Community. 2020. Available at: https://www.davincisurgerycommunity.com/group/onlinecomm unity Accessed on 19 Dec 2020
7. European Academy of Robotic Colorectal Surgery. 2020. Available at: http://first.fchampalimaud.org/en/newsroom/earcsbased-champalimaud-foundation-view/?p=. Accessed on 19 Dec 2020

8. British Association of Urological Surgeons (2015) British Association of Urological Surgeons (BAUS) Robotic surgery curriculum-guidelines for training. BAUS, London

9. Szold A, Bergamaschi R, Broeders I et al (2015) European Association of Endoscopic Surgeons (EAES) consensus statement on the use of robotics in general surgery. Surg Endosc 29:253-288

10. Society of American Gastrointestinal and Endoscopic Surgeons (2007) SAGES/MIRA consensus document on robotic surgery. SAGES, Los Angeles

11. Miskovic D, Ahmed J, Bissett-Amess R, Gómez Ruiz M, Luca F, Jayne D, Figueiredo N, Heald RJ, Spinoglio G, Parvaiz A, European Academy for Robotic Colorectal Surgery (EARCS) (2019) European consensus on the standardization of robotic total mesorectal excision for rectal cancer. Colorectal Dis 21(3):270-276. https://doi.org/10.1111/codi.14502

12. Kerr R (2019) The future of surgery. RCS Bull 101(7):264-267

13. The Joint Committee on Surgical Training. 2020. Available at: https://www.jcst.org. Accessed on 19 Dec 2020

14. Agha R, Abdall-Razak A, Crossley E, Dowlut N, Iosifidis C, Mathew G, for the STROCSS Group (2019) The STROCSS 2019 guideline: strengthening the reporting of cohort studies in surgery. Int J Surg 72:156-165

15. O'Brien BC, Harris IB, Beckman TJ, Reed DA, Cook DA (2014) Standards for reporting qualitative research: a synthesis of recommendations. Acad Med 89:1245-1251

16. Andolfi C, Umanskiy K (2017) Mastering robotic surgery: where does the learning curve lead us? J Laparoendosc Adv Surg Tech A 27:470-474

17. Carpenter BT, Sundaram CP (2017) Training the next generation of surgeons in robotic surgery. Robot Surg 4:39-44

18. Bolger JC, Broe MP, Zarog MA, Looney A, McKevitt K, Walsh D, Coffey JC (2017) Initial experience with a dual-console robotic-assisted platform for training in colorectal surgery. Tech Coloproctol 21(9):721-727. https://doi.org/10.1007/ s10151-017-1687-8

19. Jimenez-Rodriguez RM, Rubio-Dorado-Manzanares M, D1'azPavón JM et al (2016) Learning curve in robotic rectal cancer surgery: surgery: current state of affairs. Int J Colorectal Dis 31(12): $1807-1815$

20. Guend H, Widmar M, Patel S et al (2016) Developing a robotic colorectal cancer surgery program: understanding institutional and individual learning curves. Surg Endosc 31(7):2820-2828

21. National Bowel Cancer Audit. 2020. Available at: https://www. nboca.org.uk/content/uploads/2020/01/NBOCA-2019-V2.0.pdf. Accessed on 19 Dec 2020

22. Khadhouri S, Miller C, Fowler S, Hounsome L, McNeill A, Adshead J, McGrath JS, BAUS Section of Oncology (2018) The British Association of Urological Surgeons (BAUS) radical prostatectomy audit 2014/2015 - an update on current practice and outcomes by centre and surgeon case-volume. BJU Int 121(6):886-892. https://doi.org/10.1111/bju.14156

23. Graham LA, Hawn MT (2019) Learning curves and the challenges of adopting new surgical techniques. JAMA Netw Open 2(10):e1913569. https://doi.org/10.1001/jamanetworkopen. 2019.13569

24. Hall ME, Reddy RM (2017) Should every medical student receive exposure to robotic surgery? J Robot Surg 11:375-376

25. Deleon MF, Maloney Patel N (2015) R. C. Robotic colorectal surgery: outcomes and cost analysis at a single institution. In: American Society of colon and rectal surgeons annual scientific meeting MA2015 
26. Zemmar A, Lozano AM, Nelson BJ (2020) The rise of robots in surgical environments during COVID-19. Nat Mach Intell 2:566-572. https://doi.org/10.1038/s42256-020-00238-2

27. Proximie Limited. 2020. Available at: https://proximie.com. Accessed on 21 Dec 2020
Publisher's Note Springer Nature remains neutral with regard to jurisdictional claims in published maps and institutional affiliations. 